\title{
Simplified Heat Load Profile Generation
}

\author{
Clemens Felsmann, Peter Stange \\ Technische Universität Dresden \\ D-01062 Dresden, Germany \\ peter.stange@tu-dresden.de
}

\begin{abstract}
A method is presented which allows the computation of time resolved profiles of the heat load and the return temperature of buildings. The approach is primarily applied if only a few building information is available. It contains an existing data base of regression models that were developed based on empirical data. The method is described in detail followed by a discussion of the approximation quality. Exemplary results from an application are shown. Furthermore, an alternative method of the computational estimation of return temperatures involving system parameters is presented.
\end{abstract}

\section{Introduction}

Analysis and optimization of urban heat supply concepts and district heating systems contribute to energy and cost efficient heat supply. Peak heat load and annual heat demand are normally used to size the heat generators, where both load and annual heat demand are aggregated values taking into account a number of individual buildings connected to and provided by the district heating system. The knowledge of building's hourly load profiles offers the opportunity to optimize system operation including load management. In addition to that load profiles can be used to also calculate a corresponding return temperature fed back to the district heating system. So far hourly heat load profiles of buildings could be generated using the standard load profile procedures published by [BdEW] where standard load profiles of gas consumption are applied. Providing an acceptable estimation in a wide range of applications one drawback of this static approach is its inflexibility regarding the variation of external influences on the heat demand, e.g. changes of the ambient temperature or different holiday patterns. Those load profiles are based on a limited number of typical days which are usually scaled to consider the changes in demand throughout the year. This low variety may lead to an unrealistic estimation of the simultaneity of peak loads if ensembles of buildings are considered. Generation of heat load profiles using building energy simulation programs often causes some effort for model setup, parameterization and acquisition of input data. For new districts even rough building information is available only. Finally, we also know from experience that load predictions using blind simulation may differ more or less from real heat load, i.e. there is neither a practical benefit nor a need to run a detailed building simulation. Consequently, the aim is to easily generate robust heat load profiles for single buildings, ensembles of buildings or any urban subareas. There is a request to model the load based on only few parameters. Load profiles also have to be aggregated taking into account realistic simultaneity of peak loads. As ambient temperature is one of the key variables to be accounted for heat load profiles can be easily adapted by modifying annual ambient temperature profiles. Heat load pattern can be scaled to meet either building peak load or annual heat demand. The calculated full hours of use are then available for plausibility checks. The heat load generator can be applied to estimate load profiles of any consumer in a district heating network where detailed building information is not available. Beside heat load profiles also building return temperatures of the district heating connections can be estimated using a similar approach. Variable return temperatures close to reality to be applied in district heating simulations offer a more realistic feature than any other approach. Model accuracy has been analysed using evaluation metrics and several quality criteria lead to the conclusion that despite the simplified approach results are reliable and robust.

\section{Methods}

As mentioned before the aim of this approach is not a detailed building simulation that is as accurate as possible. Instead we would like to generate heat load profiles in cases where only few data is available and where it is impossible to determine the heat demand by detailed building simulation methods. The disadvantages of static concepts based on scaling a few number of profiles of typical days should be avoided. A typical approach in such a case is data driven modelling. Basically, data driven methods approximate the measured data depending on a number of known explanatory influences. Applying this model to a new combination of these explanatory data will lead to the corresponding prediction of the simulated value. Several approaches for solving this kind of problem are known and well developed. Some of the most popular ones are multiple linear regression as shown, e.g. by Fox (1997), artificial neural networks that where used by Kalogirou and Bojic (2000) for the prediction of the heat consumption of a passive solar building, Gaussian processes, see, e.g. Roberts et al. (2013) for an introduction and classical time series analysis with or without explanatory influence as presented in detail by Box, Jenkins and Reinsel (2008). In our framework multiple linear regression models have 
been used to generate the annual load profiles. Based on empirical data key variables having an impact on building heat load profiles have been isolated. Empirical heat load profiles gained in several types of buildings have been analysed. Building types have been categorized and clustered according to their specific load profile patterns. In our approach the process of model generation, database setup and model application consists of the following steps.

a) Model generation and database setup:

- Computation of the coefficients of the regression model based on empirical data.

- Adding the regression model to the database storing the regression coefficients and the key building characteristics.

b) Model application (building simulation):

- Selecting a model from the database such that it best represents the building to be simulated.

- Application of the regression model to the external influences belonging to the simulation period.

- Scaling of the resulting values to either fit the known or estimated (e.g. based on base load per unit area) installed heating power or the aggregated yearly heat demand of the considered building.

\section{Regression model}

In the following we will present the model that is used for generating heat demand profiles. A similar approach is applied for the approximation of return temperatures and will be outlined subsequently.

Since one of the main purposes is to keep the methodology of the framework easy to follow, easy to adapt and easy to distribute a method of multiple linear regression is used. To approximate the measured heat load profile $\dot{Q}_{\text {meas }}\left(t_{j}\right)$ the regression parameters $q_{i}$ in equation

$$
\dot{Q}_{\text {mod }}\left(t_{j}\right)=\sum_{i=1}^{m} q_{i} X_{\text {mod, } i}\left(t_{j}\right)
$$

are calculated such that the sum of squared residuals

$$
\epsilon_{L S Q}=\sum_{j=1}^{n}\left(\dot{Q}_{\text {meas }}\left(t_{j}\right)-\dot{Q}_{\text {mod }}\left(t_{j}\right)\right)^{2}
$$

is minimized.

In (1) $\dot{Q}_{\text {mod }}\left(t_{j}\right)$ represents the mean heating power per discrete time step $t_{j}$ with $j=1, \ldots, n$. For each of those time steps, the modelled value of heating power is represented by the weighted sum of explanatory variables $X_{\bmod , i}\left(t_{j}\right)$. In order to reduce the necessary amount of data to both generate and apply equation (1) the modelled dependency of the heat load $\dot{Q}_{\bmod }\left(t_{j}\right)$ is restricted to its key influences:

- base load (e.g. domestic hot water generation or any process heat demand),

- outside temperature (due to heat losses of the building forced by temperature difference between indoor and ambient),

- type of day (e.g. weekend, free days and working days),

- time of day (e.g. night and day).
One of the explanatory variables $X_{\bmod , i}\left(t_{j}\right)$ is a constant term which represents the base load valued by $q_{1}$. As far as there is a domestic hot water demand that is included in the load profile it is part of this base load. For most of the buildings there is a significantly different heat demand comparing working days and free days. This dependency is modelled by an explanatory variable taking binary values that indicates the two different types of days. Obviously there is a strong dependency between ambient temperature and heat load. This dependency is usually characterized by the temperatures corresponding to the heating limit and by the maximal heating power. The resulting nonlinear behaviour is represented in regression model (1) by multiple explanatory variables based on transformations of the outside air temperature, e.g. $\vartheta_{\mathrm{amb}}^{2}$. Finally, the main influencing factor to the heat demand is the time of the day. This factor mainly causes the characteristic shape of daily profiles which may include, e.g. morning and afternoon peaks and night setbacks. Due to this nonlinear dependency it is not suitable including the time as an explanatory variable in (1). Instead in a first step the measurement data is categorized respecting the time of day. In a second step for each of those categories a separate regression model consisting of the same kind of explanatory variables is calculated. Using a time discretization of 15 minutes the following model results:

$$
\dot{Q}_{\bmod , \tau}=\sum_{i=1}^{m} q_{i, \tau} X_{\bmod , i, \tau},
$$

where $\tau=1, \ldots, 96$ denotes the time frame of the day $(\tau=1: 0: 00-0: 15 \mathrm{am}, \tau=2: 0: 15-0: 30 \mathrm{am}, \ldots)$. Equation (2) allows different regression parameters belonging to the same explanatory variables but to different time frames. This property significantly improves the model accuracy since most of the dependencies differ in their quantity respecting the time of day.

After data acquisition the only task for model building is the computation of the regression parameters $q_{i, \tau}$. These parameters are finally stored in a database.

A similar approach of multiple linear regression is used to approximate the return temperature $\vartheta_{\text {rt,meas }}\left(t_{j}\right)$ by

$$
\vartheta_{\mathrm{rt}, \bmod }\left(t_{j}\right)=\sum_{i=1}^{m} p_{i} Y_{\bmod , i}\left(t_{j}\right) .
$$

Compared to (1) the additional explanatory variables

- Supply temperature, and

- Heat load (measured or approximated)

are required. As in the case of heat load modelling separate models for each time frame $\tau$ of the day were created:

$$
\vartheta_{\mathrm{rt}, \mathrm{mod}, \tau}=\sum_{i=1}^{m} p_{i, \tau} Y_{\bmod , i, \tau}
$$

It turned out that (4) leads to a better approximation of the return temperature compared to formulation (3) covering the whole day in one equation.

Obviously, it is possible to refine the regression models (2) and (4), e.g. by providing further input data, by performing an advanced regression analysis of dependencies or by a time series analysis of the residual values as it is shown by Stange, Matthees and Sander 
(2018) on an example of the heat consumption of a district heating network.

But there are downsides of more complex models in the context of the proposed application. First of all, the availability of more explanatory data in the necessary resolution in time may be problematic since these data must be available for both model building (parameter estimation) and model application (simulation). This is contrary to the aim of providing an easy to use model which is applicable without detailed information not only regarding the building itself but also regarding external influences. Further, there is the possibility of an overadaption to the source data of the regression. In addition to the well known problem of over-fitting in the proposed application this may cause the effect, that the general pattern of the load profile of a specific category of buildings gets lost.

\section{Data acquisition}

The data basis for the computation of the regression coefficients consists of empirical data gained from monitoring. Most of the heat load, the supply and the return temperature data were measured within the scope of different research projects. Usually, only in a few cases, e.g. for specific customers with a high demand, this data is monitored at least hourly or even more frequent by district heating suppliers. In most cases the available data is not separated in heat load used for drinking hot water (DHW) and for space heating. Case-dependent the empirical data which is the basis of our model setup covered a period between one and three years with a time resolution between three minutes and one hour. The corresponding ambient temperature either was included in the data sets or it was taken from the closest weather station run by [DWD]. So far empirical data of 36 different buildings have been integrated into the building clustering and typing.

Comparing the typical shapes of the averaged daily, weekly and yearly heat profiles, the following nine clusters of buildings have been identified:

- Industrial buildings

- Apartement buildings

- Single-family houses

- Schools

- Commercial buildings

- Office buildings

- Universities

- Hospitals

- Hotels

This clustering turned out to be convenient in terms of consumer allocation in practical applications.

The monitored heat load data used for model building may include effects caused by manual or automatic control of the heating system. For this reason the measured demand doesn't necessarily coincide with the actual demand of the building. The choice of explanatory variables doesn't include any information about the control of the heating system. This circumstance may cause positive as well as negative effects to the approximation quality of the models that are hard to predict in general.

Figure 1 illustrates the measured and modelled heat load on the example of a hospital from our database. It shows the time series of one week in March 2013 which was modelled with a time resolution of 15 minutes. The coefficient of determination

$$
R^{2}=1-\frac{\sum_{j=1}^{n}\left(\dot{Q}_{\text {meas }}\left(t_{j}\right)-\dot{Q}_{\text {mod }}\left(t_{j}\right)\right)^{2}}{\sum_{j=1}^{n}\left(\dot{Q}_{\text {meas }}\left(t_{j}\right)-\frac{1}{n} \cdot \sum_{k=1}^{n} \dot{Q}_{\text {meas }}\left(t_{k}\right)\right)^{2}}
$$

is a popular measure for the goodness of the fit of two time series. It takes the value one in the case of a perfect fit and zero if one of the time series doesn't contain more information than the mean value of the other. $R^{2}$ is 0.899 , which is a very good value, in the case shown in Figure 1.

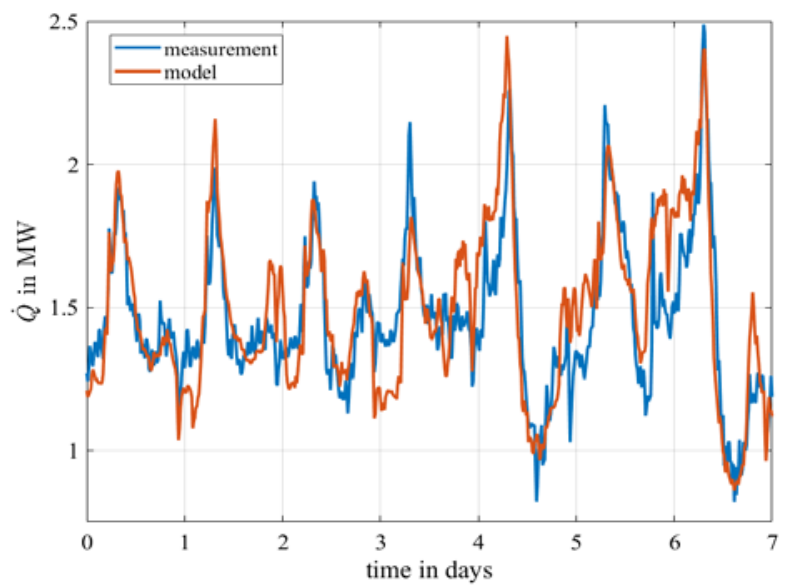

Figure 1: Comparison between the measured (blue) and the modelled (orange) heat load profile of a hospital for one week in March 2013.

In Figure 2 the approximation quality of all heat load models from our database is illustrated. The left boxplot shows the coefficient of determination $R^{2}$ corresponding to the regression models. The right boxplot shows the relative mean absolute error corresponding to the models:

$$
\epsilon_{R M A}=\frac{1}{n} \cdot \frac{\sum_{j=1}^{n}\left|\dot{Q}_{\text {meas }}\left(t_{j}\right)-\dot{Q}_{\text {mod }}\left(t_{j}\right)\right|}{\max _{j}\left|\dot{Q}_{\text {meas }}\left(t_{j}\right)\right|} .
$$

In both cases the red line highlights the median of the value. The lower and upper bounds of the blue box mark the $25 \%$ and $75 \%$ percentile, respectively. The black lines symbolizes a $99 \%$ coverage of the values if they are normally distributed. The red dots show outliers which could be an indication that for the corresponding buildings the regression model needs to be refined. Figure 2 shows, that most of the $R^{2}$ values are between 0.8 and 0.9 which represents a very good fit. This is verified by the low relative mean absolute error of less than $5 \%$ for most of the models. The outliers correspond to energy efficient buildings in the database. Obviously, the choice of explanatory variables has to be adapted for this kind of buildings, e.g. by considering solar radiation and temperature dependencies across time. 


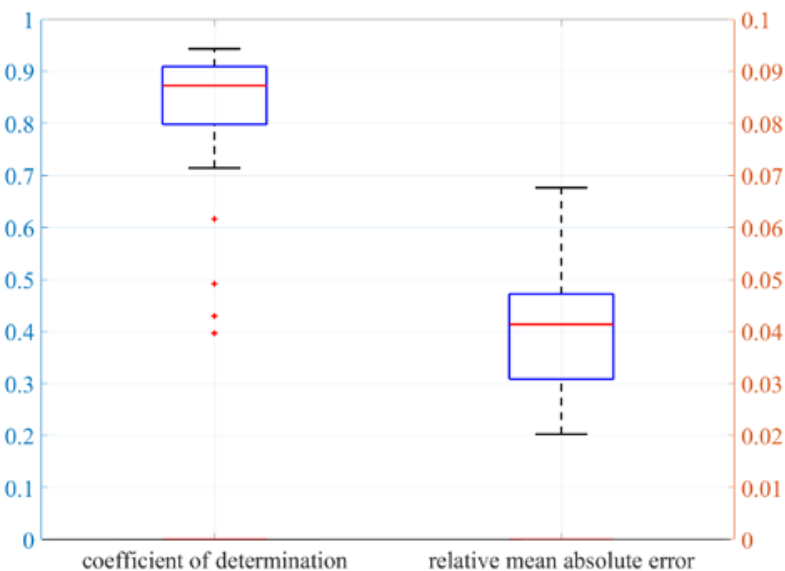

Figure 2: Evaluation criteria for the quality of the heat load models, coefficient of determination (left axis), relative mean absolute error (right axis).

Analogously, Figure 3 shows the coefficients of determination and the mean absolute errors of the return temperature models.

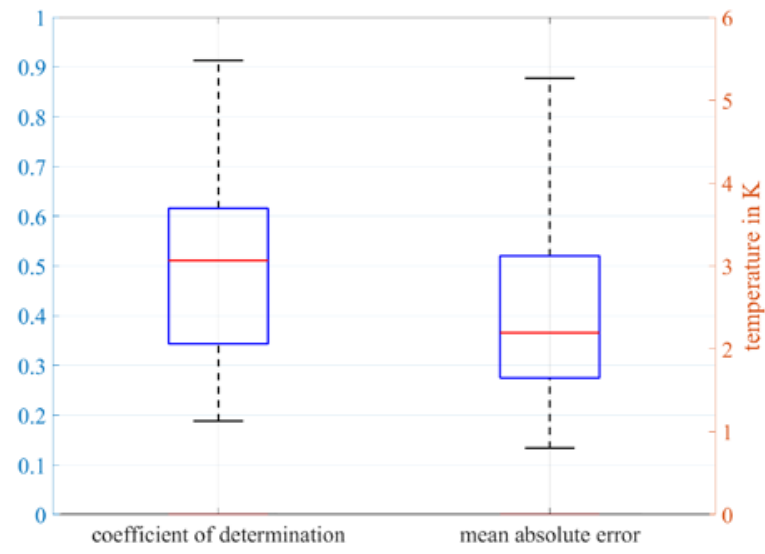

Figure 3: Evaluation criteria for the quality of the return temperature models, coefficient of determination (left axis), mean absolute error (right axis).

On one hand the values of $R^{2}$ are significantly lower compared to the heat load model. On the other hand the median of the mean absolute difference between measured and modelled return temperatures is $2.19 \mathrm{~K}$ which is rather good.

Figure 4 shows the measured and modelled return temperature on the example of an university building from our database. The time series of one week in March 2013 which was modelled with a time resolution of 15 minutes is illustrated. The coefficient of determination is $R^{2}=0.59$. The mean level of the temperature as well as the shape of the curve of measured values is fitted well. In contrast there are significant differences in the peak values. These are probably caused by external influences that are not contained in the set of explanatory variables in the regression model. Nevertheless, if no other reliable information is available this model may still be an acceptable estimation.

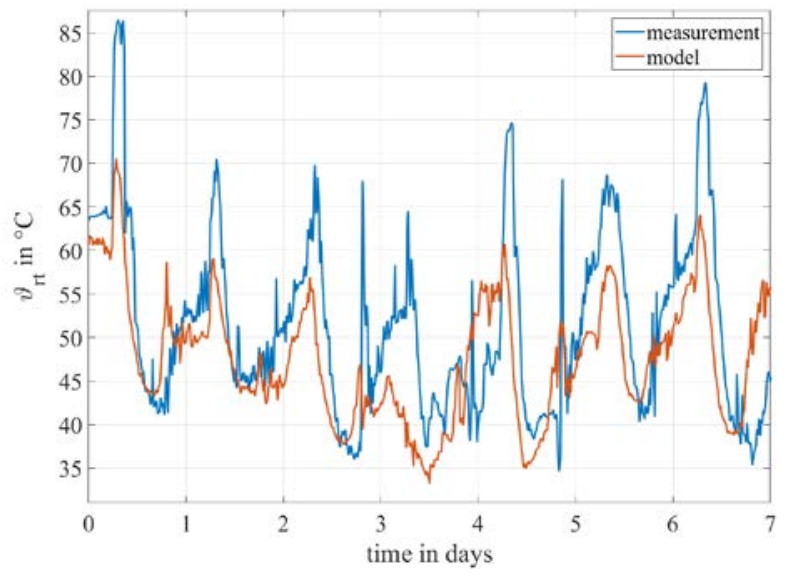

Figure 4: Comparison between the measured (blue) and the modelled (orange) profile of the return temperature of an university building for one week in March 2013.

The values of $R^{2}$ and $\epsilon_{R M A}$ shown in this chapter verify the suitability of the regression approach to model heat load profiles and return temperatures of the buildings contained in the database. Using these regression models to simulate arbitrary buildings will lead to a lower quality of approximation. Since the key applications of the proposed method are cases where only a few building information is available this inexactness can hardly be avoided by any approach.

\section{Model application}

Using the model to generate a heat load profile requires the following information:

- Type of building (to choose the appropriate model)

- Time series of ambient temperature and the type of each single day (to apply the regression coefficients)

- Peak load or heat demand in a given time intervall (to scale the resulting profile)

Based on the input the resulting heat load profile $\dot{Q}_{\text {sim }}$ is computed by combining (1) and (2). Generating a timeseries for one day with a resolution of 15 minutes leads to:

$$
\dot{Q}_{\text {sim }}=\left(\begin{array}{ccccccc}
q_{1,1} & \cdots & q_{m, 1} & & & 0 & \\
& & & \ddots & & & \\
& 0 & & & q_{1,96} & \cdots & q_{m, 96}
\end{array}\right)\left(\begin{array}{c}
X_{\text {sim }, 1,1} \\
\vdots \\
X_{\text {sim }, m, 1} \\
\vdots \\
X_{\text {sim }, 1,96} \\
\vdots \\
X_{\text {sim }, m, 96}
\end{array}\right) .
$$

This procedure can easily be adapted to time series of an arbitrary length. The resulting heat load profile corresponds in its values to the installed heating power of the building where the regression model is based on. In a final step $\dot{Q}_{\text {sim }}$ has to be scaled to match the installed heating power or annually heat demand of the considered building where (5) is applied for. Obviously, the computation of different scenarios, e.g. the variation of the ambient temperature, is performed straightforward by varying the input data.

If the return temperature needs to be computed a time series of the supply temperature must be provided additionally. Furthermore, the calculation is performed 
analogously to (5) based on equations (3) and (4) leading to:

$$
\vartheta_{\mathrm{rt}, \mathrm{sim}}=\left(\begin{array}{ccccccc}
p_{1,1} & \cdots & p_{m, 1} & & & 0 & \\
& & & \ddots & & & \\
& 0 & & & p_{1,96} & \cdots & p_{m, 96}
\end{array}\right)\left(\begin{array}{c}
Y_{\text {sim }, 1,1} \\
\vdots \\
Y_{\text {sim }, m, 1} \\
\vdots \\
Y_{\text {sim }, 1,96} \\
\vdots \\
Y_{\text {sim }, m, 96}
\end{array}\right) .
$$

Since (5) and (6) require only a small number of basic operations these models are well suited in complex district heating network simulations with a huge number of consumers as done by Paulick, Schroth, Guddusch and Rühling (2018).

\section{Alternative return temperature model}

In some applications multiple linear regression may be an unsuitable approach to simulate return temperatures. In this chapter we present an alternative method to solve this task. This approach is mainly based on system parameters which are usually known. It involves the separate estimation of both return temperature from space heating and return temperature from DHW generation finally followed by the computation of the mixing temperature. This allows the analysis of influences only related to one of both components. The effects of optimization actions, e.g. the reduction of the space heating supply temperature, can be considered. In contrast the regression based method is a black-box approach regarding the mixing temperature only. Furthermore, the variation of system parameters can't be studied using equation (6) since major changes in the domain of the explanatory variables (compared to the modelling process) will most likely lead to unreliable values due to problems caused by extrapolation.

The return temperature $\vartheta_{\text {rt,mod }}$ of the building is resulting from a mixture of the return temperatures of space heating $\vartheta_{\text {rt,H }}$ and DHW generation $\vartheta_{\text {rt,DHW }}$. It is computed in each time step by

$$
\vartheta_{\mathrm{rt}, \text { mod }}=\frac{\phi}{f_{1}} \cdot \vartheta_{\mathrm{rt}, \mathrm{H}}+\left(1-\frac{\phi}{f_{1}}\right) \cdot \vartheta_{\mathrm{rt}, \mathrm{DHW}},
$$

whereas $\vartheta_{\text {rt,DHW }}$ is assumed to be a fixed temperature known from experience depending on the type of DHW generation system. The return temperature resulting from space heating can be estimated by

$$
\vartheta_{\mathrm{rt}, \mathrm{H}}=\phi^{\frac{1}{f_{2}}} \cdot \Delta \vartheta_{\mathrm{m}, \mathrm{N}}-0.5 \cdot \Delta \vartheta_{\mathrm{N}}+\vartheta_{\mathrm{i}} .
$$

In the ideal heating curve (8) $\vartheta_{\mathrm{i}}$ denotes the set room temperature. The further temperature differences are defined as

$$
\begin{aligned}
& \Delta \vartheta_{\mathrm{m}, \mathrm{N}}=0.5 \cdot\left(\vartheta_{\mathrm{sp}, \mathrm{N}}+\vartheta_{\mathrm{rt}, \mathrm{N}}\right)-\vartheta_{\mathrm{i}} \\
& \Delta \vartheta_{\mathrm{N}}=\vartheta_{\mathrm{sp}, \mathrm{N}}-\vartheta_{\mathrm{rt}, \mathrm{N}},
\end{aligned}
$$

where $\vartheta_{\mathrm{sp}, \mathrm{N}}$ and $\vartheta_{\mathrm{rt}, \mathrm{N}}$ are the design supply and return temperatures. Furthermore, the heat load ratio $\phi$ in (7) and (8) can be computed by

$$
\phi=\max \left(0, \frac{\vartheta_{\mathrm{i}}-\vartheta_{\mathrm{amb}, \mathrm{w}}}{\vartheta_{\mathrm{i}}-\vartheta_{\mathrm{amb}, \mathrm{d}}}\right),
$$

where $\vartheta_{\mathrm{amb}, \mathrm{d}}$ is the ambient temperature in the design case and $\vartheta_{\mathrm{amb}, \mathrm{w}}$ is a weighted ambient temperature, which could be computed, e.g. as the mean value of the actual and the previous time step:

$$
\vartheta_{\mathrm{amb}, \mathrm{w}}=0.5 \cdot \vartheta_{\mathrm{amb}}\left(t_{j}\right)+0.5 \cdot \vartheta_{\mathrm{amb}}\left(t_{j-1}\right) .
$$

If $\phi=0$ the mixing return temperature $\vartheta_{\mathrm{rt} \text {,mod }}$ is equal to the return temperature $\vartheta_{\text {rt,DHW }}$ resulting from the DHW generation, i.e. there is no space heating demand and (8) needs not to be computed. Finally, equations (7) and (8) contain the two parameters $f_{1}$ and $f_{2}$ which has to be estimated, e.g. based on measured values.

The result of equation (7) can subsequently be improved by respecting further external influences providing they are known and its influence can be estimated.

\section{Results}

The presented approach was successfully used in several projects where detailed load profiles and return temperatures were unavailable and had to be generated for time-dependent analyses. One example will be outlined in the following.

\section{Example}

The method was applied to analyse a district heating network. In this case time resolved measurement data was only available at the location of the main heat generator. Regarding the consumers in the network the available data consisted of their particular annual heat demand and of the installed heating power. Furthermore, detailed measurements of supply and return temperatures were available at the location of the main generator only. In this specific application it was necessary to generate time series of the heat load and return temperatures for each of the 258 consumer located in the network. These profiles were applied to estimate the maximal heat demand for each single customer based on the aggregated yearly data that was given. Comparing these values with the actual installed heating power it is possible to identify potential incorrect supply contracts. Furthermore, the time series of heat load and return temperature were required for detailed hydraulic simulations of the district heating network.

Based on the kind of building and with respect to the actual temperature data of the year 2017 one heat load profile was generated for each customer using (5). As a first verification of the generated profiles they were aggregated and compared to the values measured at the heat generators. The additional network losses which had to be considered in this comparison were calculated by a standard approach and added to the summed load pattern of the consumers. Figure 5 shows the time series of the sum of the aggregated heat load profiles and the network losses compared to the actual measurements at the heat generator for the year 2017.

Visually the pattern of both profiles match very well. Computed on a daily basis the coefficient of determination of the heat demand profiles is $R^{2}=0.97$. Computed for the hourly values it is $R^{2}=0.86$. Regarding the few necessary information required for generating the load profile both values show the very good performance of the regression approach. 


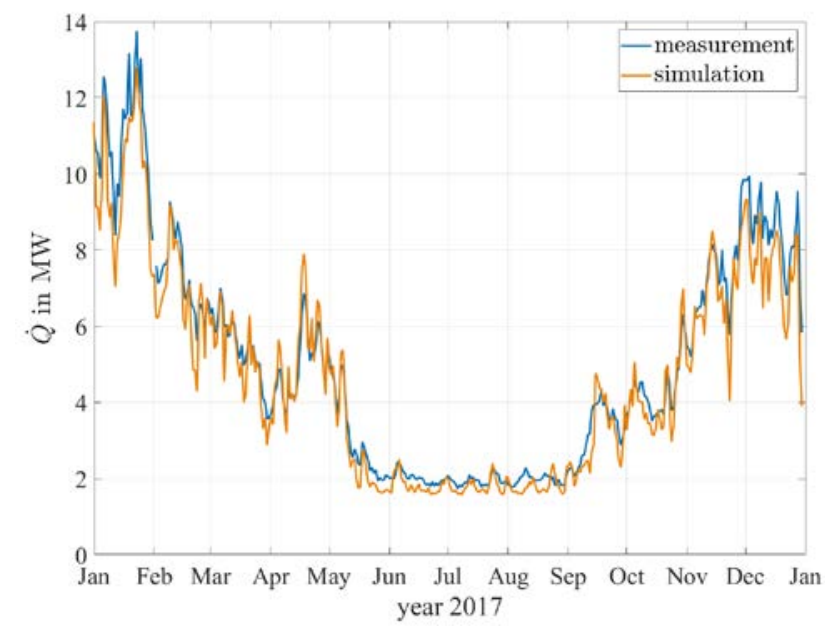

Figure 5: Comparison between actual (blue) and simulated (orange) heat load profiles of the heat generators of a district heating network.

Figure 6 illustrates the dependency between outside air temperature and heat load. Each dot represents one hourly mean value of the heating power in 2017. For both measured and modelled values the pattern looks quite similar which verifies the suitability of the regression model (2). Since the available number of building models in our database is limited the pattern of the generated values looks more radial compared to the measured data.

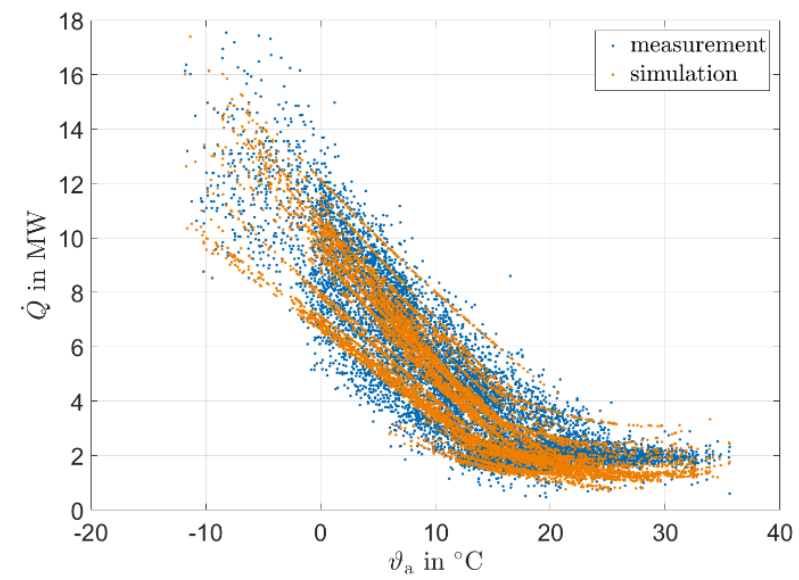

Figure 6: Temperature dependency of the heat demand. Comparison between actual (blue) and simulated (orange) data.

In Figure 7 the simulated return temperature at the location of the heat generator is compared to the measured values. The presented method (6) was applied to compute the return temperatures of the single buildings at the district heating connection. Those values were weighted by the heat load of the corresponding buildings to estimate the combined mixing return temperature at the location of the heat generator in the district heating network. The resulting values obviously differ from the measurements. Whereas in summer the return temperature is underestimated, in spring its value is slightly too high in most of the time steps. In contrast the main peak in January as well as the gradients in May and September are met very well.

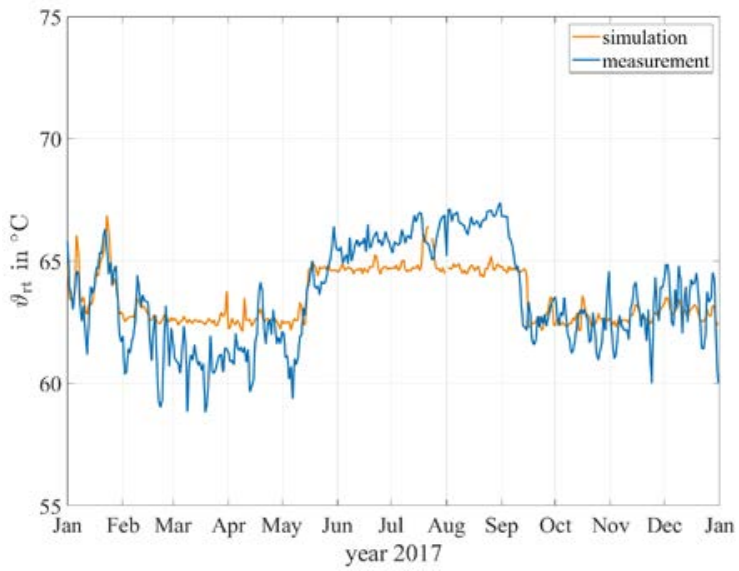

Figure 7: Comparison between actual (blue) and simulated (orange) return temperatures at the location of the heat generators.

Considering daily mean values the model is acceptable leading to a maximal absolute error of $M A E=3.63 \mathrm{~K}$. The coefficient of determination is $R^{2}=0.71$ in this case. Of course, one reason of the high approximation accuracy is that the aggregation of profiles of multiple buildings and the temporal average value in time (hourly, daily) is considered in this example. The $R^{2}$ values are probably much lower if each individual building in the district heating network is regarded separately. Unfortunately, the latter could not be quantified since no measurement data was available for the individual consumers.

\section{Conclusion}

The described regression method is applicable to generate approximated heat load profiles even without specific knowledge of the considered building and in cases with low data availability. An analogical approach is shown for generating return temperatures at the connection between building and district heating network. It can be concluded that multiple linear regression is an appropriate method for the modelling of both heat load and return temperature profiles. The method was applied analysing several district heating networks. The data verification for one of these use cases is exemplary outlined. In addition an alternative method based on system parameters for modelling the return temperature is introduced.

Based on empirical data realistic time series pattern result applying the presented methods. In contrast to standardized heat load profiles it is possible to respect varying temperature scenarios. Scaling and aggregation is easily possible. The framework can be upgraded by more detailed models.

Especially regarding energy efficient buildings the consideration of solar radiation and time dependent effects may increase the approximation quality of the heat consumption. Whereas the first can be considered by including solar radiation as an additional explanatory variable the latter can be explained by additional terms representing the auto-regression and the moving average of the heat load profile. Since data availability is a limiting factor it could be considered to use data from detailed 
building simulation as an input for setting up the regression models to increase the number of models in the database.

Using the proposed data-driven method it must be considered that extrapolation of input data may lead to unrealistic results. Furthermore, due to its simplicity this approach is not suitable to perform high precise simulations of individual specific buildings.

\section{References}

BdEW, Bundesverband der Energie- und Wasserwirtschaft e.V., https://www.bdew.de/energie/standardlastprofile-gas.

Box G.E.P., Jenkins G.M. and Reinsel G.C. (2008). Time series analysis: forecasting and control. $4^{\text {th }}$ ed. Wiley Series in Probability and Statistics, John Wiley and Sons, Inc.

DWD, Deutscher Wetterdienst, https://www.dwd.de.

Fox, J. (1997). Applied Regression Analysis, Linear Models, and Related Methods. SAGE Publications.
Kalogirou, S.A. and Bojic, M. (2000). Artificial neural networks for the prediction of the energy consumption of a passive solar building. Energy 25(5), 479-491.

Paulick S., Schroth C., Guddusch S. and Rühling K. (2018). Resulting Effects On Decentralized Feed-In Into District Heating Networks - A Simulation Study. Energy Procedia 149, 49-58.

Roberts S., Osborne M., Ebden M., Reece S., Gibson N. and Aigrain S. (2013). Gaussian processes for timeseries modelling. Philosophical Transactions of the Royal Society of London A: Mathematical, Physical and Engineering Sciences, The Royal Society.

Stange P., Matthees A. and Sander T. (2018). Operational optimization of energy systems, 25 years - established and promising use cases. Energy Procedia 149, 15-24. 